Meta

Journal des traducteurs

Translators' Journal

\title{
L'eurolecte : oui, jargon communautaire : non
}

\section{Roger Goffin}

Volume 39, numéro 4, décembre 1994

Hommage à Bernard Quemada : termes et textes

URI : https://id.erudit.org/iderudit/002930ar

DOI : https://doi.org/10.7202/002930ar

Aller au sommaire du numéro

Éditeur(s)

Les Presses de l'Université de Montréal

ISSN

0026-0452 (imprimé)

1492-1421 (numérique)

Découvrir la revue

Citer cet article

Goffin, R. (1994). L'eurolecte : oui, jargon communautaire : non. Meta, 39(4), 636-642. https://doi.org/10.7202/002930ar

\section{Résumé de l'article}

L'objet de l'étude est de démontrer que le langage utilisé dans les écrits communautaires, - ici baptisé eurolecte - ne se démarque, ni par son origine, ni par son organisation sémantique, ni par ses caractéristiques

morphosymagmatiques, ni par son fonctionnement, d'autres lectes. Replacées dans la logique des énoncés et réinscrites dans la dynamique du discours, les eurolexies permettent au contraire de décrire et de maîtriser l'afflux de notions nouvelles d'une Europe en devenir.
Tous droits réservés @ Les Presses de l'Université de Montréal, 1994
Ce document est protégé par la loi sur le droit d'auteur. L’utilisation des services d'Érudit (y compris la reproduction) est assujettie à sa politique d'utilisation que vous pouvez consulter en ligne.

https://apropos.erudit.org/fr/usagers/politique-dutilisation/ 


\title{
L'EUROLECTE : OUI, JARGON COMMUNAUTAIRE : NON
}

\author{
ROGER GOFFIN \\ Commission des Communautés européennes et \\ Université libre de Bruxelles, Bruxelles, Belgique
}

\begin{abstract}
Résumé
L'objet de l'étude est de démontrer que le langage utilisé dans les écrits communautaires, - ici baptisé eurolecte — ne se démarque, ni par son origine, ni par son organisation sémantique, ni par ses caractéristiques morphosyntagmatiques, ni par son fonctionnement, d'autres lectes. Replacées dans la logique des énoncés et réinscrites dans la dynamique du discours, les eurolexies permettent au contraire de décrire et de maîtriser l'afflux de notions nouvelles d'une Europe en devenir.
\end{abstract}

\begin{abstract}
The purpose of this study is to show that the language used in Community texts described here as "Eurolect" - is no different in origin, semantic organization or morphosyntagmatic characteristics from any other specialized dialect. On the contrary, taken in their proper context, "Eurolexemes" enable us to describe and master the flood of new concepts to which the process of building Europe has given birth - and will continue to create.
\end{abstract}

\section{UN LANGAGE MIS AU PILORI}

L'Europe communautaire — on l'a assez répété — a pris en charge sa pluralité linguistique. Son régime de neuf langues officielles est inscrit dans les tables mêmes de la loi européenne: dans les Traités, d'abord publiés en «quatre textes qui font également foi», puis élargis à neuf langues. Vecteur de la diversité et garant de l'indépendance des peuples, le multilinguisme intégral est devenu la règle d'or, la traduction le passage obligé de tout acte européen et le traducteur un maillon indispensable à l'exercice de l'autorité publique. Ce régime est conforme aux grands principes démocratiques d'une société qui recherche la transparence dans la vie de la cité et du citoyen. Jamais on n'a autant traduit, autant confronté et contrasté, en situation concrète de communication, les ressources multiples des langues. Jamais on n'a autant légiféré, jamais on n'a eu l'occasion d'étudier au quotidien comment des institutions internationales à vocation juridique agissent sur le plan langagier pour décrire et nommer des notions nouvelles dans le choc de neuf langues naturelles, enracinées dans leurs traditions juridiques, économiques, culturelles, sociales et politiques (Goffin 1990: 13-19).

Et pourtant, le langage utilisé dans les écrits communautaires fait l'objet d'attaques virulentes. Il ne cesse d'être cloué au pilori par les hommes politiques, vilipendé par les parlementaires ${ }^{1}$ et par la presse. Les censeurs nombreux et impitoyables dénoncent en français l'eurobabillage, le brouillard linguistique européen, en allemand, l'Eurowelsch ou l'Eurokauderwelsch (Kauderwelsch signifie charabia ou baragouin), en anglais, Eurospeak ou Eurofog ou usent d'un acronyme hardi composé des premières lettres officielles avant 1984 Dadefinspeaking Community $(\mathrm{da}=$ danois, $\mathrm{d}=$ deutsch, $\mathrm{e}=$ English, $\mathrm{f}=$ français, $\mathrm{i}=\mathrm{italien}, \mathrm{n}=$ néerlandais), voire l'argot du Berlaymont ${ }^{2}$. Les procureurs s'en prennent tout à la fois au vocabulaire, à la phraséologie, aux sigles des écrits communautaires pour 
en dénoncer l'ésotérisme et la pseudo-technicité. Ceux-ci véhiculeraient des notions aux contours volontairement mal définis, dissimuleraient la vérité au citoyen et $n$ 'inciteraient pas l'opinion publique à s'intéresser à l'Europe.

Il n'est pas toujours sûr que les censeurs ne visent pas plutôt la construction européenne que les mots créés pour la dire. À tout le moins font-ils porter aux mots. tous les péchés de l'Europe. Pour leur part, les économistes et les juristes, spécialistes austères du langage plutôt glacé des chancelleries nationales, reconnaissent, il est vrai sans grand enthousiasme, que les notions mises en jeu par la construction européenne devaient bien se tailler un nouvel habit linguistique.

Critiquer ce langage, s'interroger sur sa lisibilité et relever ses bizarreries n'est pas un crime de lèse-majesté. Dans une société multiculturelle, aucun langage n'est, par nature ou de jure, au-dessus de tout soupçon. Encore convient-il de blâmer avec dignité et sans se focaliser sur des données à peine vérifiées.

Ces contradictions mêmes nous obligent à nous poser plusieurs questions fondamentales. Quelle est la nature de ce langage et comment s'est-il instauré ? Quelle est son ampleur et dans quels corpus, lexicographiques ou textuels, peut-on le retrouver? Quelle est son origine et quels sont ses modes de formation?

\section{NATURE DU LANGAGE. UN LECTE, NON UN JARGON}

En donnant naissance à un ordre juridique autonome, la Communauté en construction s'est forgée un langage nouveau que l'usage a peu à peu stabilisé et consacré à mesure que se sécularisait l'idée européenne. Très tôt, les médias se sont emparés de ces termes, et, en les poussant sur le devant de la scène, ont brouillé l'image qu'ils donnent d'euxmêmes dans leur contexte juridique, politique et culturel. De plus, un nombre croissant de notions supranationales, nouvellement nommées, sont devenues des notions clefs qui se sont figées en maitres-mots, en correspondances types et en formules récurrentes, repris de texte en texte.

Est-il pour autant légitime de parler de jargon et de crier à la jargonnite? Reconnaissons que le terme jargon véhicule des contenus qui se diluent et que la terminologie linguistique ne contribue guère à en donner une image précise. Le mot jargon a pris des connotations abusivement péjoratives, à preuve J. Marouzeau (1961: 129) qui parle de «langue artificielle employée par les membres d'un groupe désireux de n'être pas compris des non-initiés» et de «terme appliqué à un parler incompréhensible à force d'être incorrect». J. Dubois (1973: 72) précise qu'il s'agit d'«une langue (surtout un vocabulaire) utilisée dans une communauté généralement marginale, qui éprouve le besoin de n'être pas comprise des non-initiés...» et d'«une langue dont on juge qu'elle est déformée, ou incorrecte, ou incompréhensible...». Le Petit Larousse (1992) assimile le terme au «langage incorrect, au charabia», mais aussi, par un retour mélioratif, à un «vocabulaire propre à une profession, à une discipline». Le Nouveau Petit Robert (1993) distingue plusieurs sens, 1. «langage déformé, fait d'éléments disparates...», 2. «(péjoratif) langage caractérisé par sa complication, l'affectation...», 3. «façon de s'exprimer propre à une profession... difficilement compréhensible». Ce sont donc presque tous des adjectifs dépréciatifs qui reviennent pour qualifier le terme, que ce soit artificiel, incorrect, déformé, corrompu, incompréhensible, ou compliqué !

Peut-on objectivement coller ces étiquettes sur le langage communautaire dont la majorité des termes, qu'ils soient ou non nouvellement forgés ou qu'ils soient utilisés dans un sens spécifique, ont fait l'objet de définitions d'ailleurs parues au Journal officiel ${ }^{3}$ ou de mises au point minutieuses de la part des juristes-linguistes? Ne faudrait-il pas plutôt admettre que ce langage malgré sa spécificité ne se démarque, ni par ses caractéristiques sémantiques, ni par ses mécanismes morphologiques et syntagmatiques, ni par ses 
constructions syntaxiques des autres lectes $^{4}$, tels que les technolectes, les chronolectes, les régiolectes, les gynolectes ou les androlectes 5 ?

L'objet de la présente étude est d'en faire la démonstration.

\section{SOURCES ET AMPLEUR DE L'EUROLECTE}

La construction de l'Europe est au principe d'une activité publicataire sans précédent. Les écrits communautaires constituent un vaste corpus de textes législatifs relevant du droit originaire et du droit dérivé, de textes administratifs liés au fonctionnement des institutions, de textes décrivant les politiques communes et ceux décrivant les politiques juridiques, sociales, culturelles et scientifiques.

Les textes du droit originaire, à savoir le Traité de Paris (1951), le Traité de Rome (1957), l'Acte unique (1987) et le Traité sur l'Union européenne, dit de Maastricht (1992) et le droit dérivé qui en découle - règlements, directives et décisions - ont acquis, par leur caractère contraignant et normalisateur, la grandeur et la dignité de textes classiques et les termes qu'ils véhiculent le statut de termes canoniques.

Pour le Traité de Paris, ce sont des termes, tels que subventions et aides (art. 4c), consultation (art. 5.2), Haute Autorité (art. 7), notification (art. 15.2), sanctions pécuniaires et astreintes (art. 36.2), prélèvement (art. 49.1), aides à la réadaptation (art. 50.1), rééducation professionnelle (art. 56.1,2), mesures conservatoires (art. 66.5). Dans le Traité de Rome et le droit dérivé, ce sont: prélèvements (agricoles), systèmes de préférences généralisées, prix de seuil, mesures d'effet équivalent, montants compensatoires monétaires, prélèvement de coresponsabilité, ressources propres. Dans l'Acte unique, ce sont principe de subsidiarité, comitologie, politiques d'accompagnement, Livre blanc, coût de la non-Europe, reconnaissance mutuelle, procédure du filet, opérateurs économiques, grand marché unique, cohésion économique et sociale. Les termes les plus récents figurent dans le Traité de Maastricht : banque centrale européenne, citoyenneté de l' Union, Conseil des Gouverneurs, fonds à finalité structurelle, fonds de cohésion, gouvernement général, acquis communautaire.

La terminologie spécifique, dans la mesure où elle existe, n'a été jusqu'ici ni recensée, ni dénombrée avec précision. Elle a certes été colligée, mais par excès. Il serait, en effet, pour le moins imprudent de conférer le statut d'européen à tel ou tel terme pour la seule raison qu'il figure, par hasard, dans un écrit communautaire ou qu'il s'y trouve actualisé. En acccordant un peu légèrement l'investiture européenne à un ensemble flou de termes de nature économique, juridique ou administrative, la plupart des publications, telles que la série des Glossaires des Communautés européennes éditée par couple de langues par le Conseil (1982), le EGW-Wörterverzeichnis (1984) publié par le Comité économique et social et le Vocabulaire de base des C.E. (1992), publié par la Commission, désorientent plutôt le linguiste qui cherche à circonscrire ce vocabulaire. Quant aux nombreux Alphabétiques consacrés aux institutions européennes - du Dictionnaire de l'Européen au $A$ Guide to the Maze en passant par Parlez-vous eurocrate? -, et qui envahissent le marché du livre, partout et dans toutes les langues, leurs auteurs y rassemblent un lot de termes du «langage communautaire» où se bousculent pêle-mêle des termes spécifiques comme subsidiarité, prix de seuil ou stabilisateur agricole, des sigles, des noms de programmes et de comités ainsi que des données biographiques et historiques sur les grands Européens!

Ces ouvrages traduisent la belle vitalité de la Communauté, sa progression quotidienne qu'ils placent souvent dans un cadre européen élargi, voire mondial. Ce sont des instruments de travail, boîtes à outils, souvent didactiques qui invitent à un parcours dans le paysage européen plutôt qu'à des analyses aux ambitions linguistiques. 


\section{ORIGINE ET MODES DE FORMATION}

Les mécanismes lexicogéniques, qui gouvernent la formation de l'eurolecte, sont pour une large part comparables à ceux que la recherche linguistique a mis en évidence pour toute production terminologique. Ces mécanismes ne se retrouvent pas, il est vrai, d'une manière égale, dans les neuf langues, chacune disposant d'une morphologie et d'une syntagmatique spécifiques qui l'obligent à privilégier l'un ou l'autre mode de formation. Toutefois, chacune des langues paraît suffisamment riche et souple pour faire face à son rythme verbal et, suivant ses modèles, aux défis néologiques d'aujourd'hui. Dans chaque langue tout peut être dit, mais tout n'a pas encore de nom.

En nous limitant au français et en grossissant les traits, nous distinguerons les catégories néologiques suivantes: (a) les néologismes de sens, (b) les néologismes de forme, (c) les néologismes syntagmatiques, (d) les métaphores, (e) les emprunts, (f) les sigles et (g) les calques.

(a) Les néologismes de sens, tels que directive, recommandation, ressources propres, règlement, appartiennent à la catégorie dont la restriction à la sphère communautaire se trouve compensée par un enrichissement du contenu sémantique; les néologismes, tels que subsidiarité, collégialité, stabilisateur, restitution, prélèvement s'obtiennent par le glissement d'une langue de spécialité à une autre.

(b) Les néologismes de forme, par dérivation affixale, tels que comitologie, surbudgétisation ou par le préfixe euro, qui se montre d'une belle vitalité dans eurobaromètre, euroguichets, euroguide, eurolaser, euromanager, europartenariat, europêche, europessimisme, et euroforteresse, ou encore eurocommunisme ou eurodollar.

(c) Les néologismes syntagmatiques se forment eux par collocation en cascade de déterminants successifs, joints ou non par un ligament, comme opération intégrée de développement, clê verte de correction, Comitê des représentants permanents, déficit démocratique, fonds d'orientation et de garantie agricole, déclaration certifiée de l'origine, double désapprobation, quatrième ressource, démonstration communautaire. Ils sont appelés à une production infinie.

(d) Les métaphores qui traversent tout vocabulaire en voie de conceptualisation, lancées et filées sans limites dans un souci d'expressivité et de plasticité.

L'adjectif vert a pris le sens de «relatif à l'agriculture» dans l'Europe verte, le taux vert (taux de conversion représentatif fixé dans le cadre de la politique agricole commune), l'écu vert (unité de compte convertie en monnaie nationale aux taux représentatifs fixés dans le cadre de la politique agricole), clé verte de correction, monnaie verte et parité verte. À l'origine, Livre vert, de couverture verte, a désigné à la Commission un document de réflexion, suivant ainsi l'usage de la procédure parlementaire britannique; ensuite, après la publication du Livre vert de 1985 sur la politique agricole commune, les médias et les organismes spécialisés ont assimilé la notion à celle de livre consacré à l'agriculture. De même, l'adjectif blanc dans Livre blanc 1985, programme sur l'achèvement du marché intérieur européen et celui de 1993 sur Croissance, compétitivité, emploi. L'adjectif bleu a connu la consécration avec l'Europe bleue, celle de la pêche; en revanche, l'Ange bleu, de la couleur de la couverture, est un recueil publié en feuillets mobiles, de la législation agricole.

Le système européen de flottement concerté des monnaies, qui distingue le flottement pur du flottement impur, a donné naissance à la métaphore du serpent communautaire (les monnaies européennes peuvent garder entre elles des marges de fluctuation interne de $1,125 \%$ soit un écart total de $2,25 \%$ ). Le miniserpent (appelé lintworm [F. ténia] en néerlandais!) a surgi au sein du Benelux où les cours des trois pays ne varient que de $1,5 \%$. On parle de limite supérieure dù serpent et 
de limite inférieure du serpent et les interventions peuvent se faire en monnaies du serpent. En matière de législation communautaire, une procédure a été baptisée filet ou normale avec filet et sa variante procédure du contre-filet.

Dans la sphère métaphorique s'inscrivent aussi des locutions évocatrices, tels que conseil jumbo, qui réunit plusieurs Conseils, horizontal, dont les compétences s'étendent à plusieurs services, transardennais, service dont une partie des Unités est implantée à Bruxelles et l'autre à Luxembourg, Bureau des mariages ou Bureau de rapprochement des entreprises, $C l u b d u$ Crocodile, du nom d'un restaurant.

(e) L'emprunt, aspect privilégié de la néologie de forme, est peu fréquent en français. C'est d'ailleurs cette langue qui sert presque toujours de langue source; tel ou tel nouveau concept est nommé dans cette langue et est «traduit» dans les autres langues, parfois par simple emprunt comme l'anglais contre-filet procedure.

(f) Véritable culte, la siglomanie règne souveraine. Acronymes et autres sigles prolifèrent pour désigner programmes, actions et systèmes ${ }^{6}$. Souvent évocateurs et mnémotechniques, les abrègements contribuent largement à donner aux textes leur caractère cryptique, par exemple, BRAIN, FAST, PIM, SPRINT ou les acronymes à fraction syllabique comme DRIVE, ÉCLAIR, CEDEFOP, CELEX, EUTERPE, EURYDICE, HERMES, NIMEXE, PROMETHEUS. Les services administratifs font preuve ici d'une belle inventivité nourrie de réminiscences mythologiques.

(g) Étant lui-même un procédé de transposition littérale, le cáqque néologique trouve dans la traduction un milieu d'extension favorable. Ici aussi, c'est le français qui tient le rôle de grande langue source et, à ce titre, imprègne et influence les autres langues au niveau lexical et sémantique. Tout en constatant l'emprise du français par le calque sur les autres langues romanes, certains linguistes l'accueillent favorablement au nom de la transparence et de l'internationalisation. D'autres, plus respectueux de la tradition linguistique que sensibles aux internationalismes, craignent que le modèle étranger ne vienne déstabiliser les structures autochtones.

\section{LE MODÈLE ORIGINAIRE DU TEXTE COMMUNAUTAIRE ET L'EUROLECTE}

Par le jeu original de ses institutions, la Communauté européenne combine, en une heureuse mixité, l'autorité intergouvernementale du Conseil européen, qui représente les États-nations, et l'autorité communautaire représentée par la Commission. Dans cette entité hermaphrodite, le processus législatif ne se révèle pas comparable à celui qui, dans les États, emprunte la voie parlementaire puisque le processus communautaire vise à établir une norme commune à plusieurs États, susceptible de concilier des traditions différentes.

Il apparaît assez vite qu'un texte communautaire prend le plus souvent appui sur un modèle national prépondérant, lequel marque de son empreinte le texte final, fruit d'un compromis, quant au fond (habitudes de pensée) et quant à la forme (habitudes de style) entre des positions et des dictions «nationales». C'est ainsi que le droit allemand et partant la diction des textes originaires ont profondément imprégné les écrits communautaires sur la concurrence. C'est ainsi que le texte du statut de la fonction publique européenne est, pour une large part, décalqué du texte français. La langue s'en ressent inévitablement. Ces textes font ainsi entrer en équilibre instable, sinon en concurrence, les termes «nationaux» façonnés par une longue tradition juridique, sociale et culturelle et les eurolexies ou euronymes qu'implique la mise en place d'une autorité communautaire supranationale. Il serait hasardeux d'affirmer qu'une coupure, factice et fâcheuse, opposerait les notions nationales transparentes dans leur évidence et les notions supranationales opaques et, pour d'aucuns, volontairement cryptées et réservées aux seuls eurocrates.

À la mesure et à l'image du texte-compromis, les versions traduites laissent transparaître en filigrane les modes d'expression du modèle, tant est forte la prégnance du texte 
originaire. Sans être asservie à ce modèle, la traduction s'en empreint de manière diffuse mais réelle, obligée de se plier, par souci d'exactitude, à ses contours et à son architecture. Cette instillation se trouve renforcée par la publication concomitante de textes, parfois segment par segment. De valeur juridique égale, les versions sont donc, du point de vue strictement linguistique, moins authentiques et moins autochtones que le modèle originaire. Comme, à la Commission, le français tient souvent le rôle de langue originaire, les traductions se glissent dans le moule du texte pré-construit quand bien même sa diction originelle serait tempérée par un travail «transnational». Il ne faut donc pas faire porter au seul traducteur le poids de la tâche qui vise à conférer aux textes des qualités littéraires de clarté et de sobriété.

Compte tenu de ce modèle sous-jacent, il faut du temps pour apprendre à déchiffrer un texte communautaire et à pouvoir reconnaître, en filigrane, la facture originaire et les indices dont la version finale est porteuse. L'examen des moutures successives d'un même texte permettrait d'en reconstituer la genèse «philologique».

\section{LISIBILITÉ ET TRANSPARENCE}

Assurer aux Européens une législation communautaire plus concise et compréhensible, rédigée d'une «plume plus légère» (J. Delors), dans une langue d'accès plus aisé, est un souhait explicitement formulé dans les Conclusions de la Présidence, au sommet d'Edimbourg, le 12 décembre 1992. Celles-ci n'hésitent pas à décrire les deux démarches possibles: la consolidation officieuse et la codification officielle qu'elles distinguent avec netteté.

L'appel à la transparence des écrits «officiels» ne date pas d'aujourd'hui. L'hermétisme dans lequel se drapent les écrits administratifs et juridiques a été dénoncé de tout temps avec véhémence. Démosthène dans son discours contre Timocrate, composé en 352 av. J.-C., prescrivait déjà la rédaction de lois en une langue compréhensible pour le peuple. Aujourd'hui, tous les pays, au nom de la souveraineté populaire et du respect de l'administré, rompent une lance en faveur de la lisibilité du discours juridique et administratif. Les Anglais prônent le plain English, les Allemands la bürgernahe Verwaltungssprache, les Danois se targuent d'une anti-ping-og-pamper tradition (ping =chic, pamper $=$ rond de cuir).

Nombreux sont les chantres qui vantent les vertus d'un langage sobre, recyclé au plus près du parler actuel, loin des circonlocutions acrobatiques et de la langue de bois. Prônant un style classique et limpide et rêvant de l'âge d'or d'une diction dépouillée que les scripteurs modernes n'auraient pas préservée, ils mènent assez paradoxalement sur la langue une réflexion normative et prescriptive à une époque de créativité langagière débordante sinon tumultueuse. L'appel à la pureté originelle et le souhait d'une véritable transparence au service de l'administré se font entendre un peu partout, et non exclusivement pour la langue prétendûment «eurocratique». Toutefois, cette politique de clarté d'expression et de sobriété se heurte à une tradition d'écriture solidement ancrée au nom de la précision de l'écrit administratif.

Si l'on s'accorde à reconnaître la spécificité de la construction européenne appelée à créer un ordre juridique nouveau dans le respect des traditions nationales, il faut admettre du mêrne coup que le langage qu'elle se forge et qu'elle manie doit être replacé dans le processus d'intégration en cours. Qu'il donne lieu à la création de mots nouveaux (euronymes) pour désigner des concepts en devenir ou qu'il transmette des notions et des désignations enracinées dans chacune des langues protagonistes (hétérolexies), ce langage est constitué d'un vaste ensemble d'énoncés qui fonctionnent normalement - nous avons tenté de le montrer - comme un sous-système au sein d'une langue et non pas 
comme une langue artificielle, ni un jargon, forme dégradée ou corrompue, d'une langue «nationale», elle-même claire et dépouillée.

Par sa nature, ses origines, ses modes de formation et son fonctionnement, ce langage - auquel on peut conférer la dignité d'eurolecte - ne se démarque aucunement des règles qui gouvernent toute langue de spécialité. Une fois replacées dans la logique des énoncés, réinsérées dans leur contexte cognitif et réinscrites dans la dynamique du discours dans laquelle elles fonctionnent, les unités lexicales des écrits communautaires, les eurolexies, prennent des allures moins ésotériques, leurs signes se révèlent souvent être plus motivés, au sens linguistique, et plus transparents, si l'on prend en considération qu'ils émanent d'une pratique sociale de communication qui, au-delà de la convergence des économies et des politiques, respecte la variété des dimensions humaines et des valeurs culturelles.

\section{Notes}

1. Voir la Question écrite $n^{\circ} 524 / 79$ de Lord 0'Hagan à la Commission, parue au J.O. C 275/18 du 31.10 .1979 et la Question écrite $\mathrm{n}^{\circ} 1753 / 86 \mathrm{du}$ même parlementaire, parue au J.O. C 339/20 du 31.12.1986.

2. Du nom du bâtiment principal de la Commission, aujourd'hui délaissé pour cause d'insalubrité. À l'origine institution catholique pour jeunes filles de bonne famille!

3. Voir à ce propos $\mathrm{H}$. Zerwes (1992) : Glossaire de référence des définitions légales mentionnées dans le Journal officiel des C.E. (1983-1992), Comité économique et social. Cette compilation présente 1375 mots clés relevés dans les textes communautaires avec indication de la source.

4. Imhasly, B, Marfurt, B. et P. Portmann : Konzepte der Linguistik, Wiesbaden, Athenaion, «Gemeint sind mit Lekten sprachliche Variationen, die eine Reihe von grundlegenden Ähnlichkeiten syntaktischer oder phonologischer Art haben, die es erlauben, sie als Teile eines übergeordneten Systems — der Sprache (zu betrachten); andererseits unterscheiden sie sich in einer großen Anzahl von Eigenschaften, die sie in ihrer jeweiligen Art sofort erkenntlich werden lassen», p. 206. Il cite Dialekte, Soziolekte, Chronolekte.

5. Tournier, J.: «L'expression euphémique des tabous». L’auteur écrit: «il parâit exagéré de considérer qu'il existe un gynolecte et un androlecte distincts.» (p. 174)

6. Nous renvoyons à ce propos à la Liste des programmes, actions, projets et systèmes d' informations communautaires (1993 : 5), Commission, Bruxelles.

\section{RÉFÉRENCES}

BUDD, S. A. (1987) : The EEC. A Guide to the Maze, London, Kogan.

CENECO (1991): Dixeco de l'Europe, Paris, Dunod.

CLOOS, J., REINESCE, G., VIGNE, D. et J. WEYLAND (1993) : Le Traité de Maastricht, Genèse, analyse et commentaires, Bruxelles, Bruylant.

CRAMPTON, Stephen (1992): Eurospeak Explained, London.

DOUTRIAUX, Y. (1992): Le Traité de l'Union européenne, Paris, Colin.

DUBOIS, Jean (1973) : Dictionnaire de linguistique, Paris, Larousse.

EG-Wörterverzeichnis (1984) : français-allemand, Bruxelles, CES.

European Communities Glossary (1990) : français-anglais. Publication Conseil CE.

Europese Almanak (1992-1993) : Europese Commissie Bureau.

FONTAINE, Pascal et Henri MALOSSE (1991): Les institutions européennes, Paris, Retz.

GOFFIN, Roger (1990): «L'Europe en neuf langues : champ d'affrontements et ferment d'intégration linguistique», Meta, 35-1, pp. 13-19.

GONTRAND, François (1991): Parlez-vous eurocrate?, Paris, Éd. Organisation.

LOPEZ de SILANES, J. P. (1988) : Diccionario de las Comunidades Europeas, Madrid, Ed. Pirámides.

MAROUZEAU, J. (1961) : Lexique de la terminologie linguistique, Paris, Geuthner.

PAXTON, John (1986) : Dicionario das Comunidades Europeias, Bruxelas.

ROUSSELET, M. et F. CHATAGNER (1991) : 300 questions tests sur la CEE, Alleur, Marabout.

SVALDI, A. (1977) : Terminologia comunitaria, Roma, Istituto di tecnica e propaganda agraria.

THØGERSEN, Niels (1988): 700 Ord om EF, publication communautaire.

VISINE, François (1987) : Dictionnaire de l'Européen, Paris, Visine.

Vocabulaire de base de la C.E. (1992) : anglais-français-chinois, Publication CE, Commission.

WEIDENFELD, Werner et Wolfgang WESSELS (1991) : Europa von A-Z, Bonn, Europa Union Verlag. 